\title{
Transverse acoustic phonon anomalies at intermediate wave vectors in $\mathrm{MgV}_{2} \mathrm{O}_{4}$
}

\author{
T. Weber,,${ }^{1,2, *}$ B. Roessli, ${ }^{3}$ C. Stock,${ }^{4}$ T. Keller,${ }^{5,2}$ K. Schmalzl, ${ }^{6}$ F. Bourdarot, ${ }^{7}$ R. Georgii, ${ }^{2}$ R. A. Ewings, ${ }^{8}$ \\ R. S. Perry, ${ }^{8,9}$ and P. Böni ${ }^{1}$ \\ ${ }^{1}$ Physik-Department E21, Technische Universität München (TUM), James-Franck-Strasse 1, 85748 Garching, Germany \\ ${ }^{2}$ Heinz-Maier-Leibnitz-Zentrum (MLZ), Technische Universität München (TUM), Lichtenbergstrasse 1, 85747 Garching, Germany \\ ${ }^{3}$ Laboratory for Neutron Scattering and Imaging (LNS), Paul Scherrer Institute (PSI), 5232 Villigen PSI, Switzerland \\ ${ }^{4}$ School of Physics and Astronomy and Centre for Science at Extreme Conditions, \\ University of Edinburgh, Edinburgh EH9 3FD, United Kingdom \\ ${ }^{5}$ Max-Planck-Institut für Festkörperforschung, Heisenbergstrasse 1, 70569 Stuttgart, Germany \\ ${ }^{6}$ Jülich Centre for Neutron Science (JCNS), Forschungszentrum Jülich GmbH, Outstation at Institut Laue-Langevin, \\ Boîte Postale 156, 38042 Grenoble Cedex 9, France \\ ${ }^{7}$ Département de Recherche Fondamentale sur la Matière Condensée, SPSMS/MDN, CEA Grenoble, 38054 Grenoble, France \\ ${ }^{8}$ ISIS Pulsed Neutron and Muon Source, STFC Rutherford Appleton Laboratory (RAL), \\ Harwell Campus, Didcot, Oxon, OX11 OQX, United Kingdom \\ ${ }^{9}$ London Centre for Nanotechnology and UCL Centre for Materials Discovery, University College London, \\ 17-19 Gordon Street, London WC1H OAH, United Kingdom
}

(Received 10 June 2017; revised manuscript received 15 October 2017; published 7 November 2017)

\begin{abstract}
Magnetic spinels (with chemical formula $A X_{2} \mathrm{O}_{4}$, with $X$ a $3 d$ transition metal ion) that also have an orbital degeneracy are Jahn-Teller active and hence possess a coupling between spin and lattice degrees of freedom. At high temperatures, $\mathrm{MgV}_{2} \mathrm{O}_{4}$ is a cubic spinel based on $\mathrm{V}^{3+}$ ions with a spin $S=1$ and a triply degenerate orbital ground state. A structural transition occurs at $T_{\mathrm{OO}}=63 \mathrm{~K}$ to an orbitally ordered phase with a tetragonal unit cell followed by an antiferromagnetic transition of $T_{N}=42 \mathrm{~K}$ on cooling. We apply neutron spectroscopy in single crystals of $\mathrm{MgV}_{2} \mathrm{O}_{4}$ to show an anomaly for intermediate wave vectors at $T_{\mathrm{OO}}$ associated with the acoustic phonon sensitive to the shear elastic modulus $\left(C_{11}-C_{12}\right) / 2$. On warming, the shear mode softens for momentum transfers near close to half the Brillouin zone boundary, but recovers near the zone center. High resolution spin-echo measurements further illustrate a temporal broadening with increased temperature over this intermediate range of wave vectors, indicative of a reduction in phonon lifetime. A subtle shift in phonon frequencies over the same range of momentum transfers is observed with magnetic fields. We discuss this acoustic anomaly in context of coupling to orbital and charge fluctuations.
\end{abstract}

DOI: 10.1103/PhysRevB.96.184301

\section{INTRODUCTION}

Magnetically frustrated and orbitally degenerate materials are of high interest to study the coupling between the lattice, spin, and orbital degrees of freedom [1-3]. One important class are the spinels, i.e., minerals possessing the chemical formula $\mathrm{A}^{2+} \mathrm{B}_{2}^{3+} \mathrm{X}_{4}^{2-}$ where $\mathrm{A}$ and $\mathrm{B}$ are divalent and trivalent metallic cations, respectively, and in most cases $\mathrm{X}$ are oxygen ions. The anions form a face-centered cubic lattice with 32 ions in the unit cell. The interstices of the close-packed structure consist of 8 tetrahedral and 16 octahedral sites which-in a normal spinel — are occupied by the smaller A and B cations, respectively (Fig. 1). The ions occupying the tetrahedral sites constitute a diamond lattice and the ions in the octahedral sites form a corner-sharing pyrochlore lattice, which is an archetype for geometrically frustrated magnetic systems [4].

In spinel vanadates- $\mathrm{A}^{2+} \mathrm{V}_{2}^{3+} \mathrm{O}_{4}^{2-}$ - the vanadium ion is situated in a octahedral environment surrounded by oxygen ions. The crystal field of the oxygen ions splits the fivefold degenerate $d$ orbitals into $e_{g}$ and the $t_{2 g}$ states with the $e_{g}$ orbitals lying higher than the $t_{2 g}$ orbitals. The vanadium ions possess two $3 d$ electrons that are distributed over the three degenerate $t_{2 g}$ orbitals, namely $d_{x y}, d_{x z}$, and $d_{y z}$. This

\footnotetext{
*tobias.weber@tum.de
}

distribution of electrons results in one hole which can occupy any of the three degenerate $t_{2 g}$ orbitals resulting in a threefold orbital degeneracy. This orbital occupancy introduces an additional spin-orbit coupling term to the Hamiltonian [5,6].

Because of the cooperative Jahn-Teller effect [7,8] for materials with an orbital degeneracy, a structural phase transition accompanied by orbital ordering $[9,10]$ sets in for $\mathrm{MgV}_{2} \mathrm{O}_{4}$ below a temperature $T_{\mathrm{OO}}$. For $T<T_{\mathrm{OO}}$ one of the crystallographic axes-which is conventionally taken along $c$-compresses with $c / a=0.9941<1$, lowering the point group symmetry at the $\mathrm{V}$ sites from cubic to tetragonal. The compression along a single axis corresponds to the $C_{11}-C_{12}$ stability condition of the elastic constants $C_{i j}$ for cubic crystals [11]. Consequently, the triplet degeneracy of the $t_{2 g}$ orbital is partially lifted. One of the vanadium electrons occupies the energetically lower-lying $d_{x y}$ orbital, while the other electron is shared among the degenerate $d_{x z}$ and $d_{y z}$ orbitals. The $d_{x y}$ orbitals on the pyrochlore lattice order in a way that their lobes point to neighboring vanadium ions in the $a b$ plane, along [110] and [110] directions [12]. The occupation of the $d_{x z}$ and $d_{y z}$ orbitals is an unresolved problem arising from the competition of three different interaction mechanisms [13]. Namely the coupling of the orbital to the elastic strain via the cooperative Jahn-Teller effect, the KugelKhomskii interaction between neighboring vanadium ions, and the spin-orbit coupling. Depending on which interaction is 


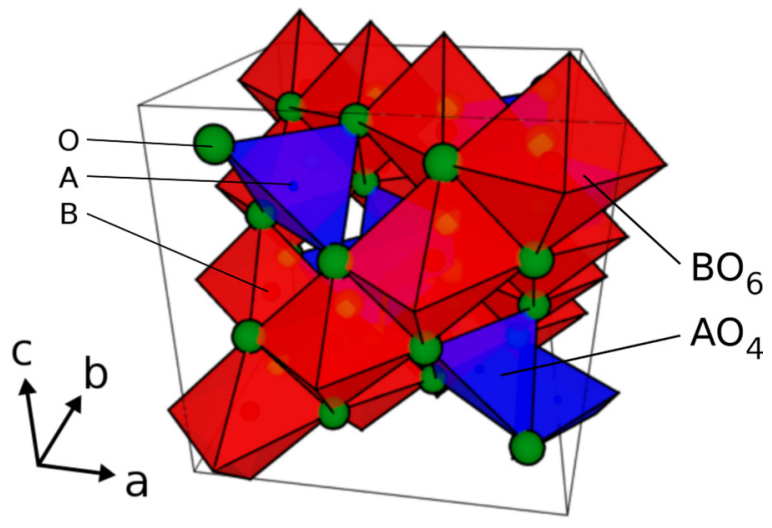

FIG. 1. Conventional unit cell of a spinel. In an $\mathrm{AB}_{2} \mathrm{O}_{4}$ spinel, the $\mathrm{A}$ metal ions are in a tetrahedral $\mathrm{AO}_{4}$ environment that constitutes a diamond lattice, and the $\mathrm{B}$ metal ions form edge-sharing $\mathrm{BO}_{6}$ octahedra arranged in a pyrochlore lattice.

assumed to dominate, different orbital ordering patterns are obtained.

A prominent theoretical model, given by Tsunetsugu and Motome [14], is based on the Kugel-Khomskii interaction between the vanadium ions, and results in a ferro-orbital (antiferro-orbital) ordering of the $d_{x y}\left(d_{x z}, d_{y z}\right)$ orbitals along the [110] ([101], [011]) directions in the pyrochlore lattice. Because this theory has been found to predict a symmetry for the structural lattice which is incompatible with measurements [15], a different model was devised by Tchernyshyov [12], treating the spin-orbit interaction to be dominating, followed by the Jahn-Teller, and - on the weakest scale-the Kugel-Khomskii interaction. It predicts an orbital ordering where one of the electrons on each vanadium ion occupies a complex superposition of $d_{x z}$ and $d_{y z}$. For both models $[12,14]$, the spin of the vanadium electron in the $d_{x y}$ orbital orders antiferromagnetically along chains in the [110] and [1 $\overline{1} 0]$ directions on the pyrochlore lattice. In the $b c$ and ac planes, an up-up-down-down spin pattern forms below the Néel temperature $T_{N}<T_{\mathrm{OO}}$. The magnetic moments point along the $c$ axis of the crystal and a strong (weak) magnetic coupling is obtained for perpendicular (parallel) chains. In the model by Tchernyshyov [12], geometric frustration of the spins in the perpendicular chains on the pyrochlore lattice gives rise to two (plus two time-reversed) degenerate ground states.

Neutron inelastic scattering and diffraction experiments by Wheeler et al. [15] found a mixture of both of these models, a real and a complex superposition of the $d_{x z}$ and $d_{y z}$ orbitals, to best reproduce their experimental data for $\mathrm{MgV}_{2} \mathrm{O}_{4}$. The interplay between lattice and spin/orbital ordering was studied in $\mathrm{MgV}_{2} \mathrm{O}_{4}$ by Watanabe et al. [16] using ultrasound measurements. They found that the $C_{11}$ and $C_{44}$ elastic constants show a softening region for cooling towards $T_{\mathrm{OO}}$ and a large discontinuity at $T_{\mathrm{OO}}$, which indeed suggests that there is a strong coupling between the orbitals of the Jahn-Teller ions and the lattice strain. In addition, Watanabe et al. [16] found a sensitivity of $C_{11}$ and $C_{44}$ on external magnetic fields up to $7 \mathrm{~T}$ in the [110] direction for the softening region near $T>T_{\mathrm{OO}}$ in contrast to $\left(C_{11}-C_{12}\right) / 2$, which does not depend on field.
In this work we present results of inelastic neutron scattering experiments above and below the orbital-ordering temperature $T_{\mathrm{OO}}$ that yield the dispersion of the acoustic phonon branches in $\mathrm{MgV}_{2} \mathrm{O}_{4}$, thus complementing the $q \rightarrow 0$ results of Watanabe et al. [16]. Unlike ultrasound measurements which probe acoustic fluctuations in the $\lim _{q \rightarrow 0}$ and Raman spectroscopy which is a strictly $q=0$ probe, neutron spectroscopy can investigate excitations associated with a uniform lattice deformation at all wave vectors, and hence wavelengths, over the entire Brillouin zone. We focus our measurements on the acoustic phonons and not the optical phonons previously reported to exist for energies above $\sim 10 \mathrm{meV}$ using optical techniques $[17,18]$. We will show that there exists a range of acoustic phonon wavelengths associated with the $\left(C_{11}-C_{12}\right) / 2$ elastic constant where the TA mode both softens in energy and also increases in linewidth indicative of decreased lifetime.

This paper is divided into three main sections including this Introduction. We first describe the neutron scattering experiments studying the low energy acoustic phonons where the softening in energy over intermediate wave vectors and then the linewidth broadening indicative of a shortening of phonon lifetimes is described. We finally conclude with a discussion comparing our results to theories for soft modes in Jahn-Teller systems and also a comparison between the anomalies observed here and in metallic systems with acoustic instabilities.

\section{EXPERIMENTS}

\section{A. Sample characterization}

Three cylindrical single crystals of $\mathrm{MgV}_{2} \mathrm{O}_{4}$, each of about $2 \mathrm{~cm}$ height and $0.75 \mathrm{~cm}$ diameter, were grown using a mirror image furnace. Characterization was performed using bulk susceptibility measurements and neutron diffraction confirming the presence of both a magnetic and structural transition. The bulk measurements of the heat capacity and the magnetic susceptibility show well defined structural and antiferromagnetic transitions at temperatures of $T_{\mathrm{OO}} \approx 63 \pm 1 \mathrm{~K}$ and $T_{N} \approx 42 \mathrm{~K}$, respectively, demonstrating the high quality of the crystals. Using neutron diffraction on the single crystals, the space group was confirmed to be face-centered cubic $F d \overline{3} m$ above $T_{\mathrm{OO}}$ and tetragonal $I 4_{1} / a$ below $T_{\mathrm{OO}}$ [19].

At the neutron diffractometer MIRA [20,21], the temperature $(T)$ dependence of the lattice constants was measured using $\theta-2 \theta$ scans around the (220) Bragg reflections of the single crystals. The volume of the unit cell was determined to be $V \approx 593 \AA^{3}$. The very weak $T$ dependence of $V$ (Fig. 2) is compatible with the results of Refs. [15,22].

\section{B. Phonon dispersion}

We first describe the softening in energy of the acoustic fluctuations in $\mathrm{MgV}_{2} \mathrm{O}_{4}$. Inelastic neutron scattering experiments were performed using the triple-axis spectrometer (TAS) EIGER [23] at the spallation source SINQ [24] at the Paul Scherrer Institute (PSI) in Villigen, Switzerland and the IN22 [25] triple-axis spectrometer at the Institute Laue-Langevin (ILL) in Grenoble, France. Both spectrometers were operated in the constant final energy mode with 

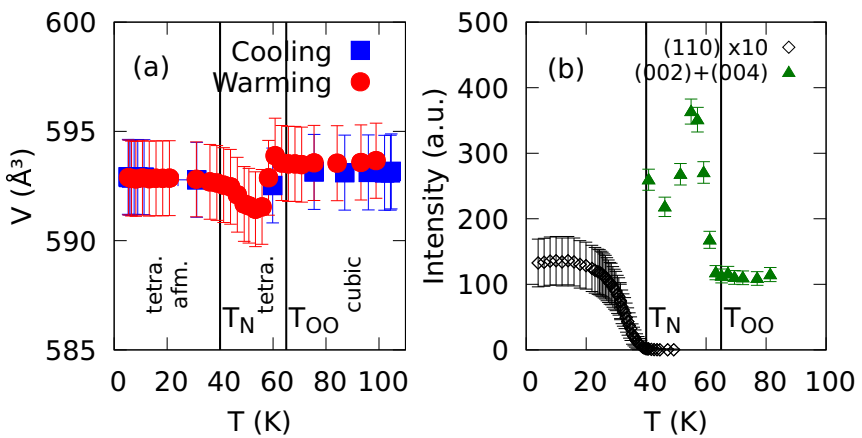

FIG. 2. (a) Temperature-dependent change of the unit cell volume $V$ as determined using neutron diffraction. Note that the error bars include the instrument resolution as systematic error. (b) The intensities of the $\vec{Q}=(110)$ and the $\vec{Q}=(004)$ Bragg reflections as a function of temperature illustrating the structural transition at $T_{\mathrm{OO}}=63 \mathrm{~K}$ and magnetic ordering temperature of $T_{N}=42 \mathrm{~K}$. The data in (b) was taken using the RITA spectrometer in two-axis mode with $E_{i}=5 \mathrm{meV}$. The (004) Bragg peak was measured at the (002) position using $\lambda / 2$ with the scattered Be filter removed. Note that the intensity of (110) has been magnified by a factor of 10 relative to the (004) data.

$E_{f}=14.68 \mathrm{meV}$. At EIGER and IN22, the dispersion of the acoustic phonon branches was measured using a vertically focusing monochromator and horizontally focusing analyzer without using any collimation. For the EIGER measurements, crystals oriented in the $h k 0$ and the $h h l$ planes were used, collecting data around the (400) and (440) Bragg peaks. For IN22 we used the $h k 0$ scattering plane.

In addition, experiments at small reduced momentum transfer $q$ were conducted using the cold TAS option of MIRA [20,21] at the MLZ in Garching, Germany, using neutrons with fixed incident energies $3.78<E_{i}<4.97 \mathrm{meV}$ yielding an energy resolution (full-width at half-maximum, FWHM) of $0.11<\Delta E<0.17 \mathrm{meV}$. At MIRA, a vertically focusing monochromator was combined with a flat analyzer. No collimation was installed in the incident and scattered beams. Higher order neutrons were removed by means of a cooled beryllium filter. At MIRA, we used the $h k 0$ scattering plane and collected data in the (220) Brillouin zone.

Furthermore, we employed the time-of-flight spectrometer (TOF) MERLIN [26] at the Rutherford Appleton Laboratory in Harwell, UK. MERLIN uses a multirepetition-rate chopper, where we selected incident energies of $E_{i}=24 \mathrm{meV}$ and $E_{i}=49 \mathrm{meV}$ and used a Fermi chopper frequency of $350 \mathrm{~Hz}$. Data were collected with the sample rotation covering the full range of $90^{\circ}$ around the (440) Bragg reflection and the crystal oriented in the $h h l$ plane. Analysis was performed using the software HORACE [27].

The primary goal of our experiments was to establish the dispersion of the TA1, TA2, and LA phonon modes. Note that in our notation, the elastic constant $C_{44}$ is related to the sound velocity of the twofold degenerate transverse mode TA1 propagating along a [001] direction with a polarization along [100] or [010]. $\left(C_{11}-C_{12}\right) / 2$ corresponds to the TA2-mode propagating along [110] with a polarization along [1 $\overline{1} 0]$. $\left(C_{11}+C_{12}+2 C_{44}\right) / 2$ is the sound velocity of the LA-phonon propagating along [110]. Owing to the neutron cross section



FIG. 3. Typical data for transverse phonons TA1 and TA2 are shown for $T=10 \mathrm{~K}$ and $T=80 \mathrm{~K}$. There is a clear softening of the TA2 mode in the cubic phase when compared with the low temperature tetragonal phase.

and selection rules associated with phonon eigenvectors [28], sensitivity to both the TA1 and TA 2 phonon modes is obtained with triple-axis measurements when the crystal is aligned in the $h k 0$ scattering plane, while alignment in the $h h l$ plane only affords measurements of the TA 1 phonon. Time-of-flight measurements using chopper spectrometers allow momentum transfers out of the horizontal scattering plane to be measured and therefore afford sensitivity to both TA1 and TA2.

Typical data of TA1 and TA2 phonons are shown in Fig. 3 for temperatures $T=10 \mathrm{~K}$ (tetragonal phase) and $T=80 \mathrm{~K}$ (cubic phase). In Figs. 3(a) and 3(b) example (4q0) (TA1) and $(4-q, 4+q, 0)$ (TA2) phonon data from IN22 are shown. Figures $3(\mathrm{c})$ and $3(\mathrm{~d})$ depict typical $(44 q)$ (TA1) and $(4-q, 4+q, 0)$ (TA2) slices from MERLIN. The TA1 mode does not show any $T$ dependence, while the TA2 mode becomes softer in energy in the high temperature cubic phase. We selected the example $q$ values shown in Fig. 3 as they are in the region where the effect is strongest and where the peaks can be clearly separated from other contributions, e.g., incoherent scattering. Figure 4 summarizes the results of all phonon measurements that were conducted at various temperatures in the range $10 \leqslant T \leqslant 200 \mathrm{~K}$. A transverse optic phonon branch is visible at $E \approx 20 \mathrm{meV}$ near the zone boundary. Attempts using both triple-axis and time-of-flight measurements failed to track this mode closer to the zone center.

The dispersion of the LA and TA1 phonon branches do not show appreciable changes between the cubic and the tetragonal phases. In contrast, the TA 2 branch exhibits an observable $T$ dependence. At intermediate $q$ values, the dispersion shows a "spoonlike" anomalous behavior, i.e., the phonons soften in 


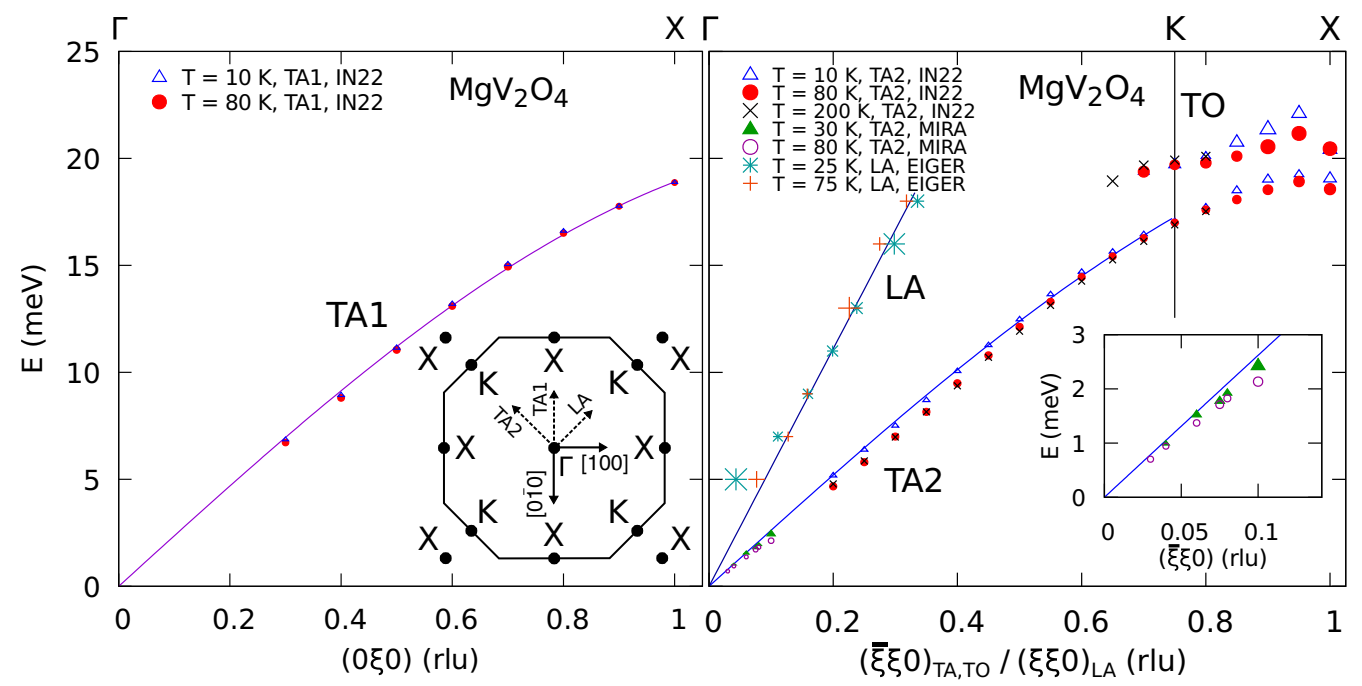

FIG. 4. The dispersion of the TA1 and the LA modes is independent of $T$ when going from the tetragonal to the cubic phase. When comparing the cubic $(T>63 \mathrm{~K})$ to the tetragonal $(T<63 \mathrm{~K})$ phonon branches, the TA2 modes show a spoonlike behavior at intermediate $q$ values. The sizes of the symbols indicate the sizes of the error bars. The inset in the left panel shows the $h k 0$ plane of the face-centered cubic Brillouin zone and the scan directions. The inset in the right panel shows a magnification of the low- $q$ TA2 phonon branch.

energy when entering the cubic phase for $T>T_{\mathrm{OO}}$. While the data are suggestive of a softening in energy of the TA2 phonon near the zone center, the effect is not as large as at intermediate wave vectors. The effect at small $q$ close to the zone center $\Gamma$ is also less pronounced than the $q \rightarrow 0$ behavior reported by Watanabe et al. [16] using ultrasound sensitive to acoustic fluctuations on the $\mathrm{MHz}$ time scale.

The detailed temperature dependence of two TA2 phonons at $q=(\overline{0.075} 0.0750), q=(\overline{0.3} 0.30)$, and $q=\left(\overline{\frac{1}{2}} \frac{1}{2} 0\right)$ are shown in Fig. 5. Both the modes at small and large $q$ soften by $\sim 0.1$ and $0.4 \mathrm{meV}$, respectively, when entering the cubic phase. For $T_{\mathrm{OO}}<T<200 \mathrm{~K}$, the low- $q$ phonons harden by $\sim 0.2 \mathrm{meV}$, while the large- $q$ modes further soften by $\sim 0.2 \mathrm{meV}$.

\section{Phonon linewidths}

Having established the presence of softening over intermediate wave vectors of the TA2 acoustic phonon, the linewidth indicative of the phonon lifetime is now discussed. We have determined the linewidth of phonons by analyzing the inelastic scattering data from EIGER and IN22 using the dynamical structure factor given by Ref. [29] which obeys detailed balance required for neutron cross sections

$$
\begin{aligned}
S(q, E)= & \frac{S_{0}}{|A \sin (x q)| \pi}\left[\frac{1}{\exp \left(E / k_{B} T\right)-1}+1\right] \\
& \times\left(\frac{ \pm \Gamma_{p}}{[E \mp|A \sin (x q)|]^{2}+\Gamma_{p}^{2}}\right) .
\end{aligned}
$$

Here the dispersion of the TA2 branch is modeled with a simple sine function that is appropriate to describe acoustic phonons. The line shape of the phonons is approximated with a Lorentzian function. The reduced momentum transfer is given by $q=2 \pi \sqrt{\left(h^{2}+k^{2}\right) / a^{2}+l^{2} / c^{2}}$, where $a$ and $c$ are the lattice constant defining the tetragonal unit cell. The term in square brackets is the Bose occupation factor for temperature $T$ and energy $E . \Gamma_{p}$ denotes the half-width at half-maximum of the phonon, $S_{0}$ is an overall scaling factor. For $\Gamma<q<K$ the TA2 dispersion at low temperature (Fig. 4) can be approximated by $A=(22.5 \pm 0.1) \mathrm{meV}$ and $x=(0.825 \pm 0.004)$. Here $A$ and $x$ serve as scaling factors for the energy and reduced momentum of the dispersion, respectively.

$S(q, E)$ as given by Eq. (1) was convoluted with the EckoldSobolev resolution function [30] of a TAS spectrometer using Monte Carlo integration. This novel algorithm was used because it reproduces the resolution function of focusing TAS better than the algorithm of Ref. [31]. Fitting the data was performed using Minuit's simplex minimiser [32]. A full description of the software tool Takin that was developed by some of the authors and used for the convolution fits can be found in Refs. [33,34].

The $q$ dependence of $\Gamma_{p}$ of the TA2 branch is shown in Fig. 6. While $\Gamma_{p}$ is essentially $p$ independent at $10 \mathrm{~K}$, it attains a maximum about halfway from the $\Gamma$ point to the $K$ point at $80 \mathrm{~K}$. The maximum occurs at the $q$ position where we also observe the strongest softening of the TA2 phonon branch (Fig. 4). No appreciable changes were observed for the other acoustic phonon branches or for the linewidth of the TA2 phonons in the tetragonal phase.

The temperature dependence of the TA2 phonons at $q=$ $\left(\frac{1}{2} \frac{1}{2} 0\right)$ and $q=(\overline{0.4} 0.40)$ are shown in Fig. 7. $\Gamma_{p}$ increases when warming the sample from $T=10 \mathrm{~K}$ towards the Néel transition at $T_{N} \approx 40 \mathrm{~K}$. Here $\Gamma_{p}$ seems to saturate at $\approx 200 \mu \mathrm{eV}$ before increasing again to a maximum value $\Gamma_{p} \approx 450 \mu \mathrm{eV}$ in the vicinity of the structural phase transition $T_{\mathrm{OO}} \approx 63 \mathrm{~K}$.

Note that the linewidth at $q=(\overline{0.4} 0.40)$ was determined with high accuracy by means of the neutron-resonance spinecho (NRSE) technique at the triple-axis spectrometer TRISP at MLZ [35]. TRISP was set up in a negative-negative-positive scattering configuration. The NRSE coils were aligned such that the focusing condition for the TA2 branch was fulfilled. The data were corrected for resolution effects reducing 

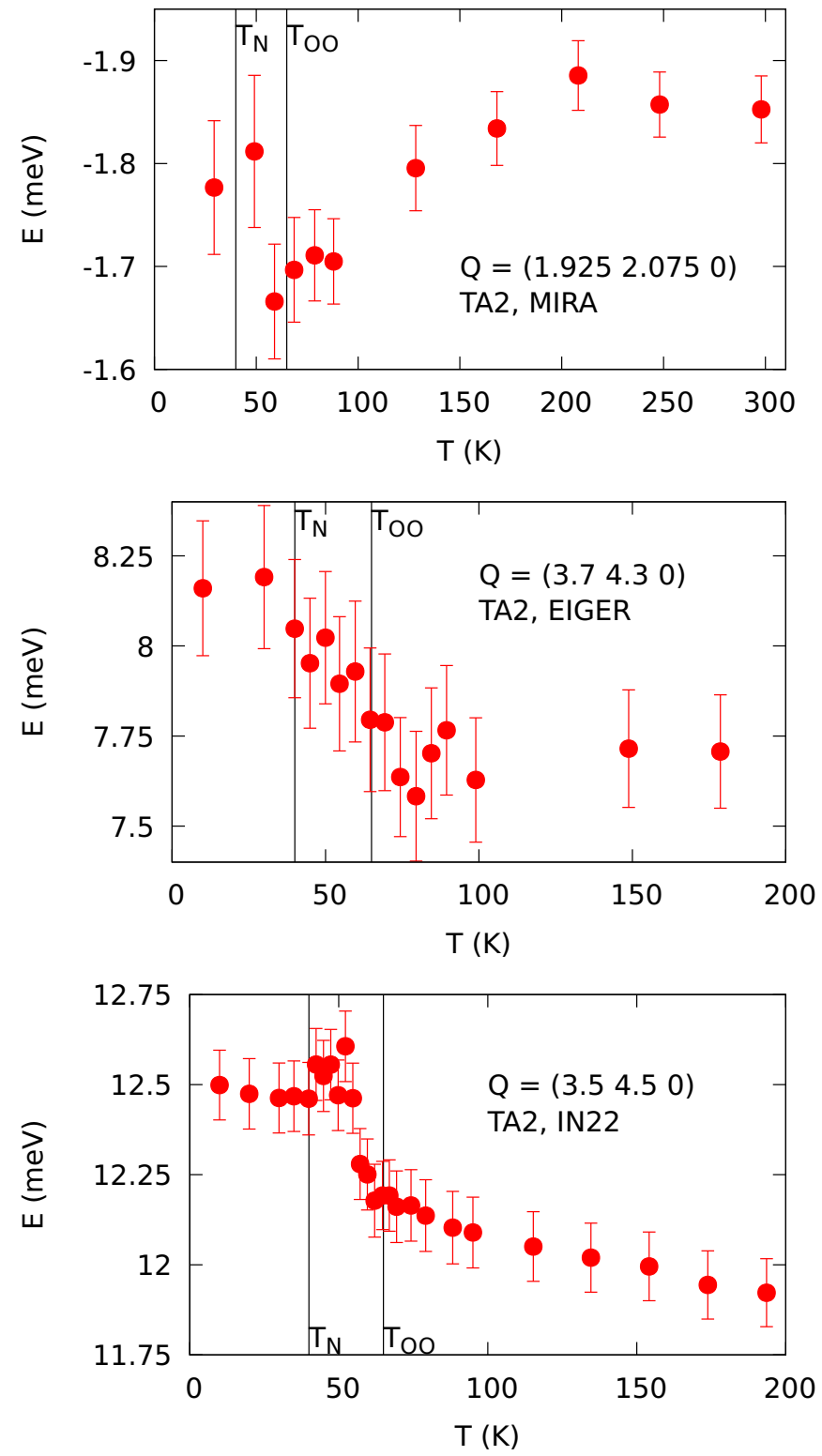

FIG. 5. The dispersion of the TA2 phonons softens and hardens at $q=(\overline{0.075} 0.0750)$ (top) and $q=(\overline{0.5} 0.50)$ (bottom), respectively, when approaching the cubic-tetragonal phase transition near $T_{\mathrm{OO}}=$ $63 \mathrm{~K}$ from high temperatures. For $q=(\overline{0.3} 0.30)$ (middle) there is no change in phonon energies in the $T>T_{\mathrm{OO}}$ region. Please note that at MIRA we measured using neutron energy gain $(-E)$ for reasons of analyzer efficiency and angular constraints at the instrument. The physics of the system is unaffected by this choice.

polarization [36,37]. These are caused by the sample mosaic and the slope of the phonon dispersion.

\section{Field dependence of phonon energy}

We finally present the magnetic field dependence of the transverse acoustic phonon lifetimes and energies in the same wave vector region where temperature dependent anomalies are observed. While a magnetic field response has been reported for polar phonons in spinels based on optical data [38], magnetic field effects on the acoustic phonon response have been reported in superconductors and also in metals where

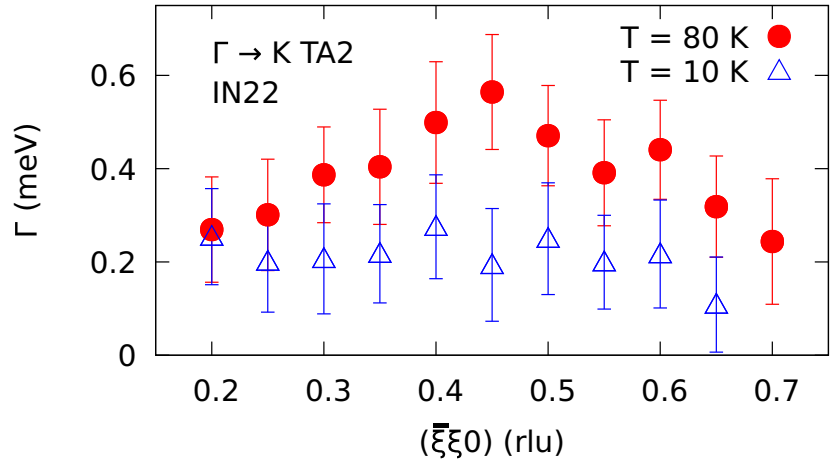

FIG. 6. Linewidth $\Gamma_{p}$ of the TA2 phonon branch. In the tetragonal phase at $10 \mathrm{~K}$ (blue triangles), $\Gamma_{p}$ is independent of momentum transfer. In the cubic phase at $T=80 \mathrm{~K}$ (red points), $\Gamma_{p}$ attains a maximum halfway from the $\Gamma$ to the $K$ point.

the Fermi surface topology is relatively flat in momentum space [39]. Attempts on semiconducting materials have failed to observe any effect [40].

The field dependence of $\Gamma_{p}$ of the TA2 phonon at $q=$ ( $\overline{0.4} 0.40$ ) was determined for $\mathrm{MgV}_{2} \mathrm{O}_{4}$ by application of a magnetic field $B_{[001]}$ along the [001] direction (Fig. 8). The phonon energy $E$ is independent of $B_{[001]}$ in the cubic phase while $E$ decreases subtly at $T=40 \mathrm{~K}$ in the tetragonal phase above $7 \mathrm{~T}$ by $\approx 0.2 \mathrm{meV}$ up to the highest magnetic field achievable in this experiment. The results are suggestive of a slight decrease in energy of the acoustic phonon at high magnetic fields at intermediate wave vectors. Further measurements to higher fields and also in other orbitally degenerate spinels would be helpful for establishing this effect.

\section{DISCUSSION}

By measuring the phonon dispersion along the highsymmetry directions $[0 \xi 0]$ and $[\bar{\xi} \xi 0]$ using neutron scattering, we have demonstrated an anomaly in the TA2 branch along the $\langle\xi \xi 0\rangle$ directions associated with the structural and orbital phase transition in $\mathrm{MgV}_{2} \mathrm{O}_{4}$ at $T_{\mathrm{OO}}$ (Fig. 4). An energy

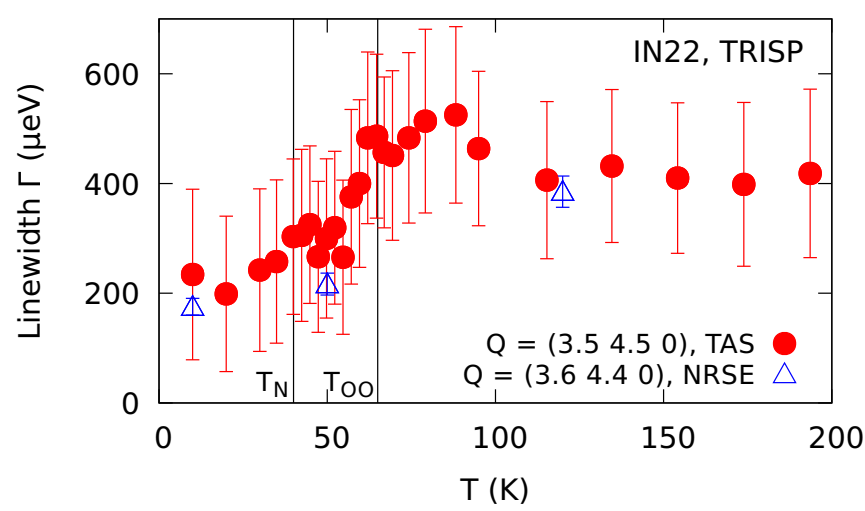

FIG. 7. Temperature dependence of the TA2 phonons at $Q=$ (3.5 4.5 0) using conventional TAS spectroscopy (red dots) and $Q=$ (3.6 4.4 0) using TRISP (blue triangles). A subtle plateau can be identified between $T_{N}$ and $T_{\mathrm{OO}} . \Gamma_{p}$ increases to approximately $0.45 \mathrm{meV}$ in the cubic phase where it remains constant up to $T=200 \mathrm{~K}$. 

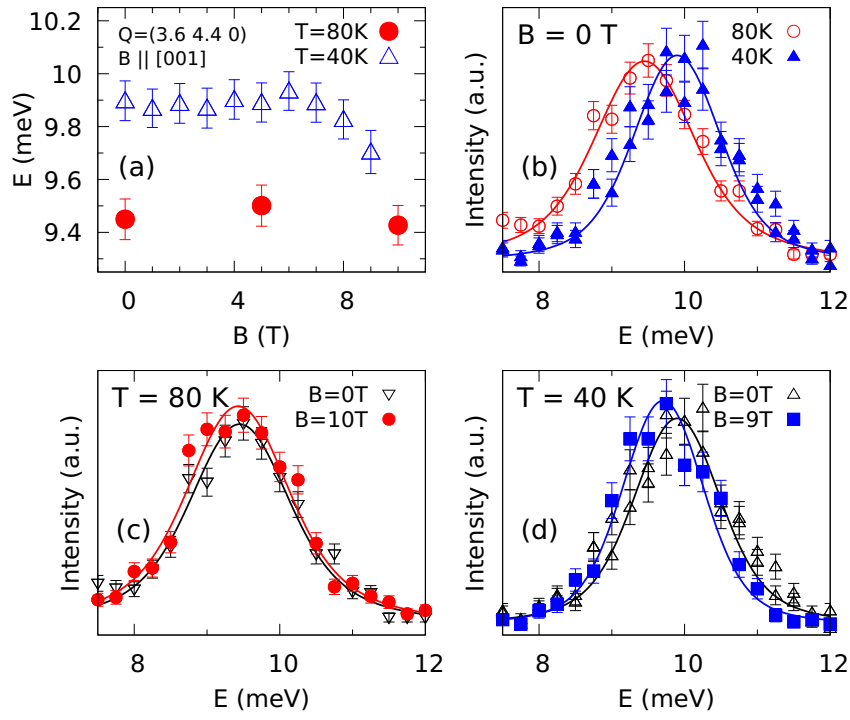

FIG. 8. (a) The field dependence of the TA2 phonon at the $q$ position ( $\overline{0.4} 0.40)$. (b)-(d) Depict selected phonon data. A small softening of $\approx 0.2 \mathrm{meV}$ is observed at high fields in the tetragonal phase for $T=40 \mathrm{~K}$ as shown by blue triangles in (a) and (d).

softening at intermediate wave vectors midway between the $\Gamma$ and the $K$ point is observed with increasing temperature. This softening leads to a spoonlike dispersion of the TA2 branch.

We first consider the possibility of thermal expansion [41] of the lattice as an explanation for the acoustic phonon anomalies in $\mathrm{MgV}_{2} \mathrm{O}_{4}$. A change in the unit cell volume would result in a change in the size of the Brillouin zone and, assuming a fixed zone boundary energy, would give a corresponding shift in phonon velocity. This cannot explain our data for two reasons. First, the phonon anomaly is only observable in $\mathrm{MgV}_{2} \mathrm{O}_{4}$ over a range of intermediate wave vectors and no change in the $\lim _{q \rightarrow 0}$ slope is observed using neutron scattering on the $\mathrm{THz}$ time scale. Second, the change in phonon energy is larger then would be expected from such an effect and is inconsistent with the thermal expansion data presented in Fig. 2 and also from the higher resolution $\mathrm{x}$-ray data for the unit cell volume presented in Refs. [22,42]. Therefore, the anomaly observed here is not due to thermal changes in the lattice constants.

The phonon anomaly in $\mathrm{MgV}_{2} \mathrm{O}_{4}$ measured with neutron scattering differs from the response observed with ultrasound [43]. At $T_{\mathrm{OO}}$, Watanabe et al. [16] reported a temperature dependent change of the sound velocity of the TA2 mode $\Delta v \approx 25 \%$. We find no observable change for momentum transfers near the zone center, however we measure a spoonlike anomaly for intermediate wave vectors. We speculate that the difference between the neutron low- $q$ data and the ultrasound data in the $\lim _{q \rightarrow 0}$ originates from the differing energy and length scales of the two measurements. Owing to temporal resolution, neutron scattering measure fluctuations on the $\mathrm{THz}$ time scale while ultrasound measures the dynamic response for long wavelengths typically on the $\mathrm{MHz}$ time scale. Similar differences between neutron scattering and ultrasound have previously been reported for spinels in Ref. [44]. The neutron scattering results do not reflect an observable softening of the TA2 phonon on the THz time scale, rather an anomaly over a range of intermediate wave vectors of $\Delta v \approx(12 \pm 5) \%$.

It is interesting to compare our results with theoretical predictions of soft acoustic phonons near a Jahn-Teller distortion. Considering the Jahn-Teller effect and coupling to acoustic phonons, a theoretical model for the phase transition from the tetragonal to the cubic phase is given in papers by Pytte $[45,46]$. In the first paper, Pytte presents a theory which models the temperature dependence of the elastic constants as a result of the coupling between the elastic strain tensor and the twofold degenerate $d$ orbitals. Including higher-order anharmonic coupling, the $T$ dependence of the transverse acoustic phonon mode propagating along [110]

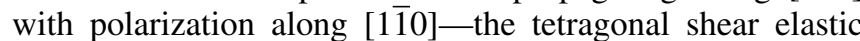
mode-shows a softening with decreasing temperature, a step discontinuity at the transition temperature $T_{\mathrm{OO}}$, followed by an increase of the velocity of sound for further decreasing temperature. The second paper [46] includes the coupling of the degenerate orbitals to the optical phonon modes and finds a "central peak" in the dynamical structure factor $S(q, \omega)$. Central peaks have been reported to often co-occur with soft modes and had first been identified in $\mathrm{SrTiO}_{3}$ [47] and $\mathrm{Nb}_{3} \mathrm{Sb}[48,49]$. They are also discussed theoretically in Refs. [50,51].

The theory developed by Pytte predicts temperature dependent changes to the TA2 phonons (with velocity proportional to $C_{11}-C_{12}$ ) and this is the same mode which displays anomalies in $\mathrm{MgV}_{2} \mathrm{O}_{4}$ over intermediate wave vectors. However, there are several important differences which make the relevance of this theory to the case here questionable. The theoretical prediction is in the context of a twofold orbital degeneracy associated with the partial occupancy of the $e_{g}$ orbitals. As outlined above, this is not the case for $S=1$ $\mathrm{V}^{3+}$ where two electrons occupy the $t_{2 g}$ orbitals resulting in a threefold orbital degeneracy. Pytte [45] also predict a true soft mode whose energy reaches zero at the phase transition for momenta $q \rightarrow 0$. A general theory for transitions driven by orbital degeneracy by Elliott [52] and Young [53] also reach the same conclusion with regards to the prediction of a soft mode close to the zone center. Experimentally, this would be reflected in the neutron response by a softening of the TA2 acoustic phonon branch and is more compatible with the results from ultrasound and also in agreement with neutron scattering experiments studying the low energy acoustic phonons in Jahn-Teller active $\operatorname{PrAl}_{3}$ [54]. In contrast to these predictions and previous experimental examples for Jahn-Teller driven structural distortions, we observe an anomaly at finite $q$ over a limited intermediate range in wave vector. Another discrepancy concerns the central peak at $E=0$ [46], which we do not observe at finite $q$. The strong increase in zone center Bragg scattering upon cooling into the tetragonal phase, which had previously been reported by Wheeler et al. [15], may be instead related to the central peak, however it appears at a different $q$ than the acoustic phonon anomaly. Based on these comparisons with theory and experiment, it is difficult to associate the TA2 phonon anomaly in $\mathrm{MgV}_{2} \mathrm{O}_{4}$ with a soft mode due to a proximate Jahn-Teller distortion resulting in breaking of the ground state orbital degeneracy and a soft acoustic mode in the $\lim _{q \rightarrow 0}$. 
The simultaneous broadening and softening of an acoustic phonon over a range in wave vector transfers is indicative of coupling to some other degree of freedom (see for example discussion of coupling in Refs. [55-58]). Coupling of acoustic phonons to crystal field [59] driven distortions have been experimentally studied with examples being the TA2 phonon anomalies reported in $\mathrm{DyVO}_{4}$ and $\mathrm{TbVO}_{4}$ [60,61]. Similar coupling effects have also been discussed in $\mathrm{UO}_{2}$ [62]. These examples involve the coupling of an acoustic phonon to a crystal field level with a similar energy scale to that of the phonon and this is also predicted to be required in the case of magnetic pyrochlores [63]. While the $d d$ transitions in $3 d$ transition metal ions are large $(\sim 1-2 \mathrm{eV})$ [64-68] making them unlikely to be involved with this process, the lower energy spin-orbit split levels may have a more appropriate energy scale. As discussed by Tchernyshyov [12], the ground state of $\mathrm{V}^{3+}$ derived by diagonalizing the spin-orbit Hamiltonian is a $j_{\text {eff }}=2$ quintuplet which is separated by an energy scale of $\sim 10 \mathrm{meV}$ [6] to an excited $j_{\text {eff }}=1$ triplet state. The splitting of such degenerate ground state due to a local molecular field could provide the spin-orbit crystal field level with the correct energy scale to couple to the acoustic phonon. However, we emphasise that such a coupling in the framework of the theories described above would involve a true softening of the fluctuations near the Brillouin zone center inconsistent with the phonon anomalies in $\mathrm{MgV}_{2} \mathrm{O}_{4}$.

Another possibility is coupling between the acoustic phonons and low energy magnon excitations from the $\mathrm{V}^{3+}$ $S=1$ sites. No magnon excitations were observed to cross the phonon branch in our experiments, however, it should be noted that the comparatively large momentum transfers optimized for phonon measurements are where magnetic form factors ensure reduced neutron scattering cross sections from magnons. But, such magnetic excitations have been reported to be highly dispersive [15], gapped [69], and located in reciprocal space away from the low-energy acoustic lattice fluctuations, making coupling in the context of the theories discussed above likely weak. While full magnon dispersion curves from neutron scattering are limited in $\mathrm{MgV}_{2} \mathrm{O}_{4}$, neutron measurements in $\mathrm{ZnV}_{2} \mathrm{O}_{4}$ [70] observe a significant gap of $\sim 10 \mathrm{meV}$ at the magnetic zone center while we observe anomalies at a displaced momentum and at lower energies. Based on the energy scale in comparison to analogous systems and the different momentum transfer away from either the zone boundary or center, we conclude that coupling to magnons is unlikely.

It is interesting to compare the results in $\mathrm{MgV}_{2} \mathrm{O}_{4}$ to metallic systems with acoustic instabilities. Our results show similarities to the measurements by Weber et al. [71] who determined the dispersion of the TA phonons in a metallic manganite. In this material, a charge and orbital ordering (COO) sets in at low temperatures. Above $T_{\mathrm{COO}}$, Weber et al. [71] report a softening of the TA phonon at $q=\left(\frac{1}{4} \frac{1}{4} 0\right)$ by $\Delta E \approx 0.25 \mathrm{meV}$ accompanied by an increase in linewidth $\Delta \Gamma_{\text {HWHM }} \approx 0.25 \mathrm{meV}$. Similar anomalies have been reported in other metallic manganites on decreasing temperature [72]. The anomaly in these example manganites is associated with electron-phonon coupling for wave vectors associated with Fermi surface nesting, given the metallic electronic response. Indeed, similar intermediate acoustic phonon anomalies have been reported in a number of materials where there is a coupling between lattice and charge degrees of freedom. One example is the one-dimensional conductor TTF-TCNQ [73] which displays a softening of a longitudinal acoustic phonon over intermediate wave vectors near a Peierls transition and also in superconductors where the change in linewidth has been related to the onset of a gap in the electronic quasiparticle response [74-78]. The wave vector at which the acoustic mode is unstable in these examples is determined by the electronic Fermi surface and hence the metallic properties.

While the analogy between soft acoustic modes in metallic systems is not obvious given that $\mathrm{MgV}_{2} \mathrm{O}_{4}$ is an insulator, there are several properties that indicate that charge fluctuations may be playing a role in this spinel. There is evidence that $\mathrm{MgV}_{2} \mathrm{O}_{4}, \mathrm{ZnV}_{2} \mathrm{O}_{4}$, and $\mathrm{CoV}_{2} \mathrm{O}_{4}$ are proximate to an insulator-metallic transition under pressure evidenced through both calculations [79] and also thermodynamic and transport measurements [80]. This has led to theoretical studies indicating that both $\mathrm{MgV}_{2} \mathrm{O}_{4}$ and $\mathrm{ZnV}_{2} \mathrm{O}_{4}$ contain large charge fluctuations and should be considered in a partially delocalized regime [81]. Therefore, both theory and experiment are suggestive of electronic or charge fluctuations in $\mathrm{MgV}_{2} \mathrm{O}_{4}$ and the possible coupling between the acoustic phonons and the charge channel is further corroborated by our magnetic field measurements which would alter the chemical potential [82]. While $\mathrm{MgV}_{2} \mathrm{O}_{4}$ is an insulator, the similarity between the acoustic phonon anomaly presented above and the studies in metallic systems discussed here support the notion of charge fluctuations in $\mathrm{MgV}_{2} \mathrm{O}_{4}$.

The similarity between the $\mathrm{MgV}_{2} \mathrm{O}_{4}$ phonon anomalies and metallic systems discussed above is reminiscent of the Peierls transition in one-dimensional systems, with TTFTCNQ discussed above being an example. This analogy may be appropriate for $\mathrm{MgV}_{2} \mathrm{O}_{4}$ [83] given the one-dimensional magnetic $\mathrm{V}^{3+}$ chain interactions which have been measured using neutron spectroscopy [15]. Similar one-dimensional magnetic correlations have been reported in $\mathrm{ZnV}_{2} \mathrm{O}_{4}$ [70] and also in $\mathrm{MgTi}_{2} \mathrm{O}_{4}$ [84], both of which have a triply orbital degenerate ground state. Supporting this possibility further, spin dimerization has been reported in the spinel $\mathrm{CuIr}_{2} \mathrm{~S}_{4}$ [85] where both $\mathrm{Ir}^{3+}$ and $\mathrm{Ir}^{4+}$ are present. Given this similarity to other spinels and the one-dimensional magnetic correlations, an instability towards dimerization resulting from an "anti-Jahn-Teller" distortions has been predicted to apply to $\mathrm{MgV}_{2} \mathrm{O}_{4}$ and other orbitally degenerate V-based spinels [86], as well as the Verwey transition in $\mathrm{Fe}_{3} \mathrm{O}_{4}$ [74]. The $T_{2}$ acoustic phonon anomalies we observe in $\mathrm{MgV}_{2} \mathrm{O}_{4}$ are along the same direction as the strong magnetic exchange resulting from orbital ordering resulting from the Jahn-Teller distortion, but we emphasise the softening and dampening is most prominent in the high temperature cubic phase. While the analogy with the Peierls transition in one-dimensional systems might be compelling, to our knowledge there is no report of structural dimerization in either $\mathrm{ZnV}_{2} \mathrm{O}_{4}$ or $\mathrm{MgV}_{2} \mathrm{O}_{4}$. We speculate that the acoustic phonon anomaly in $\mathrm{MgV}_{2} \mathrm{O}_{4}$ may indicate the close proximity to such a structural phase without the actual formation of long-range structural order. It may even be indicative of spatially localized structural distortions that would increase the acoustic phonon lifetime in a similar manner to that reported in disordered piezoelectrics [87]. 
Supporting this, we note that additional structures are known to compete with the low temperature tetragonal phase [88].

In conclusion, we identify a softening and damping of the TA2 phonon modes over a range of intermediate wave vectors. Neutron measurements of the acoustic phonons at low momentum transfers do not show any observable softening on the $\mathrm{THz}$ time scale in contrast to expectations of a uniform softening of the mode due to a change in the elastic constants. The combined linewidth broadening and also softening indicates a coupling between the acoustic TA2 phonon and another degree of freedom, analogous to electron-phonon coupling observed in metallic compounds with a structural instability. The results are suggestive of coupling to charge fluctuations predicted by theory.

\section{ACKNOWLEDGMENTS}

We thank R. Schwikowski and A. Mantwill for technical support. Financial support from the EPSRC is gratefully acknowledged. This work is based on experiments performed at the Swiss spallation neutron source SINQ, Paul Scherrer Institute (PSI), Villigen, Switzerland. The project has received funding from the European Union's Seventh Framework Programme for research, technological development and demonstration under the NMI3-II Grant No. 283883. This work is based upon experiments performed at the IN22 instrument operated by JCNS at the Institute Laue-Langevin (ILL), Grenoble, France. The authors gratefully acknowledge the financial support provided by JCNS to perform the neutron scattering measurements at the Institute Laue-Langevin (ILL), Grenoble, France. This work is furthermore based upon experiments performed at the TRISP instrument operated by MPG and the MIRA instrument at the Heinz Maier-Leibnitz Zentrum (MLZ), Garching, Germany. Experiments at the ISIS Pulsed Neutron and Muon Source were supported by a beamtime allocation from the Science and Technology Facilities Council. This work was part of the Ph.D. thesis of T. Weber [89].
[1] Y. Tokura, Phys. Today 56, 50 (2003).

[2] A. J. Millis, Nature (London) 392, 147 (1998).

[3] T. Rudolf, C. Kant, F. Mayr, J. Hemberger, V. Tsurkan, and A. Loidl, New J. Phys. 9, 76 (2007).

[4] P. W. Anderson, Phys. Rev. 102, 1008 (1956).

[5] D. S. McClure, Solid State Phys. 9, 399 (1959).

[6] A. Abragam and B. Bleaney, Electron Paramagnetic Resonance of Transition Ions (Dover, New York, 1986).

[7] M. D. Kaplan and B. G. Vekhter, Phenomena in Jahn-Teller Crystals (Springer Science, New York, 1995).

[8] G. A. Gehring and K. A. Gehring, Rep. Prog. Phys. 38, 1 (1975).

[9] P. G. Radaelli, New J. Phys. 7, 53 (2005).

[10] S. K. Pandey, Phys. Rev. B 84, 094407 (2011).

[11] R. A. Cowley, Phys. Rev. B 13, 4877 (1976).

[12] O. Tchernyshyov, Phys. Rev. Lett. 93, 157206 (2004).

[13] D. I. Khomskii, Cooperative Jahn-Teller Effect and Orbital Ordering (Cambridge University Press, Cambridge, UK, 2014).

[14] H. Tsunetsugu and Y. Motome, Phys. Rev. B 68, 060405 (2003).

[15] E. M. Wheeler, B. Lake, A. T. M. N. Islam, M. Reehuis, P. Steffens, T. Guidi, and A. H. Hill, Phys. Rev. B 82, 140406(R) (2010).

[16] T. Watanabe, T. Ishikawa, S. Hara, A. T. M. N. Islam, E. M. Wheeler, and B. Lake, Phys. Rev. B 90, 100407(R) (2014).

[17] Z. V. Popović, G. De Marzi, M. J. Konstantinović, A. Cantarero, Z. Dohčević-Mitrović, M. Isobe, and Y. Ueda, Phys. Rev. B 68, 224302 (2003).

[18] S. Jung, J. Noh, J. Kim, C. L. Zhang, S. W. Cheong, and E. J. Choi, J. Phys.: Condens. Matter 20, 175205 (2008).

[19] Wheeler et al. [15] has suggested the space groups of $\mathrm{MgV}_{2} \mathrm{O}_{4}$ to be $F \overline{4} 3 m$ (cubic) and $I \overline{4} m 2$ (tetragonal) based on the measurement of weak Bragg peaks which are absent in the space group $F d \overline{3} m$.

[20] R. Georgii and K. Seemann, J. Large-Scale Res. Facilities 1, A3 (2015).

[21] R. Georgii, T. Weber, M. Skoulatos, M. Janoschek, S. Mühlbauer, C. Pfleiderer, and P. Böni, arXiv:1710.00589.
[22] H. Mamiya, M. Onoda, T. Furubayashi, J. Tang, and I. Nakatani, J. Appl. Phys. 81, 5289 (1997).

[23] U. Stuhr, B. Roessli, S. Gvasaliya, H. M. Rønnow, U. Filges, D. Graf, A. Bollhalder, D. Hohl, R. Bürge, M. Schild, L. Holitzner, C. Kaegi, P. Keller, and T. Mühlebach, Nucl. Instrum. Methods Phys. Res., Sect. A 853, 16 (2017).

[24] W. E. Fischer, Physica B: Condens. Matter 234-236, 1202 (1997).

[25] L. P. Regnault, F. Mantegazza, B. Longuet, B. Geffray, and J. Flouquet, (1999), Report ILL-RA-1998. Reference 30044523. INIS 30(39). ILL, Grenoble, France.

[26] R. I. Bewley, R. S. Eccleston, K. A. McEwen, S. M. Hayden, M. T. Dove, S. M. Bennington, J. R. Treadgold, and R. L. S. Coleman, Physica B: Condens. Matter 385-386, 1029 (2006).

[27] R. A. Ewings, A. Buts, M. D. Le, J. van Duijn, I. Bustinduy, and T. G. Perring, Nucl. Instrum. Methods Phys. Res., Sect. A 834, 132 (2016).

[28] J. Harada, J. D. Axe, and G. Shirane, Acta Crystallogr. Sect. A 26, 608 (1970).

[29] B. Fåk and B. Dorner, Physica B: Condens. Matter 234, 1107 (1997).

[30] G. Eckold and O. Sobolev, Nucl. Instrum. Methods Phys. Res., Sect. A 752, 54 (2014).

[31] M. Popovici, Acta Crystallogr. Sect. A 31, 507 (1975).

[32] I. Antcheva et al., Comput. Phys. Commun. 182, 1384 (2011).

[33] T. Weber, R. Georgii, and P. Böni, SoftwareX 5, 121 (2016).

[34] T. Weber, SoftwareX 6, 148 (2017).

[35] T. Keller and B. Keimer, J. Large-Scale Res. Facilities 1, A37 (2015).

[36] K. Habicht, T. Keller, and R. Golub, J. Appl. Crystallogr. 36, 1307 (2003).

[37] K. Habicht, R. Golub, F. Mezei, B. Keimer, and T. Keller, Phys. Rev. B 69, 104301 (2004).

[38] T. Rudolf, C. Kant, F. Mayr, J. Hemberger, V. Tsurkan, and A. Loidl, Phys. Rev. B 76, 174307 (2007).

[39] R. Pynn and J. D. Axe, J. Phys. F 4, 1898 (1974). 
[40] R. Comès, S. M. Shapiro, B. C. Frazer, and G. Shirane, Phys. Rev. B 24, 1559 (1981).

[41] A. A. Maradudin, Phys. Status Solidi B 2, 1493 (1962).

[42] R. Kaur, T. Maitra, and T. Nautiyal, J. Phys.: Condens. Matter 25, 065503 (2013).

[43] T. Ishikawa, T. Watanabe, S. Hara, A. T. M. N. Islam, E. M. Wheeler, and B. Lake, J. Phys.: Conf. Ser. 592, 012107 (2015).

[44] P. Thompson and N. W. Grimes, Solid State Commun. 25, 609 (1978).

[45] E. Pytte, Phys. Rev. B 3, 3503 (1971).

[46] E. Pytte, Phys. Rev. B 8, 3954 (1973).

[47] T. Riste, E. Samuelsen, K. Otnes, and J. Feder, Solid State Commun. 9, 1455 (1971).

[48] G. Shirane and J. D. Axe, Phys. Rev. Lett. 27, 1803 (1971).

[49] J. D. Axe and G. Shirane, Phys. Rev. B 8, 1965 (1973).

[50] B. I. Halperin and C. M. Varma, Phys. Rev. B 14, 4030 (1976).

[51] R. A. Cowley and S. M. Shapiro, J. Phys. Soc. Jpn. 75, 111001 (2006).

[52] R. J. Elliott, Physica B 86-88, 1118 (1977).

[53] A. P. Young, J. Phys. C 8, 3158 (1975).

[54] R. J. Birgeneau, J. K. Kjems, G. Shirane, and L. G. Van Uitert, Phys. Rev. B 10, 2512 (1974).

[55] R. K. Wehner and E. F. Steigmeier, RCA Rev. 36, 70 (1975).

[56] S. Wakimoto, C. Stock, Z.-G. Ye, W. Chen, P. M. Gehring, and G. Shirane, Phys. Rev. B 66, 224102 (2002).

[57] J. Hlinka, S. Kamba, J. Petzelt, J. Kulda, C. A. Randall, and S. J. Zhang, Phys. Rev. Lett. 91, 107602 (2003).

[58] C. Stassis, M. Bullock, J. Zarestky, P. Canfield, A. I. Goldman, G. Shirane, and S. M. Shapiro, Phys. Rev. B 55, R8678(R) (1997).

[59] S. Brühl, Z. Phys. B 35, 287 (1979).

[60] R. L. Melcher and B. A. Scott, Phys. Rev. Lett. 28, 607 (1972).

[61] J. R. Sandercock, S. B. Palmer, R. J. Elliott, W. Hayes, S. R. P. Smith, and A. P. Young, J. Phys. C 5, 3126 (1972).

[62] S. J. Allen, Phys. Rev. 166, 530 (1968).

[63] Y. Yamashita and K. Ueda, Phys. Rev. Lett. 85, 4960 (2000).

[64] M. W. Haverkort, A. Tanaka, L. H. Tjeng, and G. A. Sawatzky, Phys. Rev. Lett. 99, 257401 (2007).

[65] C. Kant, T. Rudolf, F. Schrettle, F. Mayr, J. Deisenhofer, P. Lunkenheimer, M. V. Eremin, and A. Loidl, Phys. Rev. B 78, 245103 (2008).

[66] M. M. V. Schooneveld, R. Kurian, A. Juhin, K. Zhou, J. Schlappa, V. N. Strocov, T. Schmitt, and F. M. F. de Groot, J. Phys. Chem. C 116, 15218 (2012).

[67] Y.-J. Kim, A. P. Sorini, C. Stock, T. G. Perring, J. van den Brink, and T. P. Devereaux, Phys. Rev. B 84, 085132 (2011).

[68] R. A. Cowley, W. J. L. Buyers, C. Stock, Z. Yamani, C. Frost, J. W. Taylor, and D. Prabhakaran, Phys. Rev. B 88, 205117 (2013).
[69] S. L. Gleason, T. Byrum, Y. Gim, A. Thaler, P. Abbamonte, G. J. MacDougall, L. W. Martin, H. D. Zhou, and S. L. Cooper, Phys. Rev. B 89, 134402 (2014).

[70] S.-H. Lee, D. Louca, H. Ueda, S. Park, T. J. Sato, M. Isobe, Y. Ueda, S. Rosenkranz, P. Zschack, J. Íñiguez, Y. Qiu, and R. Osborn, Phys. Rev. Lett. 93, 156407 (2004).

[71] F. Weber, S. Rosenkranz, J.-P. Castellan, R. Osborn, H. Zheng, J. F. Mitchell, Y. Chen, S. Chi, J. W. Lynn, and D. Reznik, Phys. Rev. Lett. 107, 207202 (2011).

[72] M. Hoesch, P. Piekarz, A. Bosak, M. Le Tacon, M. Krisch, A. Kozłowski, A. M. Oleś, and K. Parlinski, Phys. Rev. Lett. 110, 207204 (2013).

[73] G. Shirane, S. M. Shapiro, R. Comès, A. F. Garito, and A. J. Heeger, Phys. Rev. B 14, 2325 (1976).

[74] S. M. Shapiro, M. Iizumi, and G. Shirane, Phys. Rev. B 14, 200 (1976).

[75] F. Weber, A. Kreyssig, L. Pintschovius, R. Heid, W. Reichardt, D. Reznik, O. Stockert, and K. Hradil, Phys. Rev. Lett. 101, 237002 (2008).

[76] F. Weber, L. Pintschovius, W. Reichardt, R. Heid, K.-P. Bohnen, A. Kreyssig, D. Reznik, and K. Hradil, Phys. Rev. B 89, 104503 (2014).

[77] T. Keller, P. Aynajian, K. Habicht, L. Boeri, S. K. Bose, and B. Keimer, Phys. Rev. Lett. 96, 225501 (2006).

[78] M. Bullock, J. Zarestky, C. Stassis, A. Goldman, P. Canfield, Z. Honda, G. Shirane, and S. M. Shapiro, Phys. Rev. B 57, 7916 (1998).

[79] S. Blanco-Canosa, F. Rivadulla, V. Pardo, D. Baldomir, J.-S. Zhou, M. García-Hernández, M. A. López-Quintela, J. Rivas, and J. B. Goodenough, Phys. Rev. Lett. 99, 187201 (2007).

[80] A. Kismarahardja, J. S. Brooks, A. Kiswandhi, K. Matsubayashi, R. Yamanaka, Y. Uwatoko, J. Whalen, T. Siegrist, and H. D. Zhou, Phys. Rev. Lett. 106, 056602 (2011).

[81] Y. Kato, G.-W. Chern, K. A. Al-Hassanieh, N. B. Perkins, and C. D. Batista, Phys. Rev. Lett. 108, 247215 (2012).

[82] T. Maitra and R. Valentí, Phys. Rev. Lett. 99, 126401 (2007).

[83] O. Tchernyshyov, R. Moessner, and S. L. Sondhi, Phys. Rev. B 66, 064403 (2002).

[84] M. Schmidt, W. Ratcliff, P. G. Radaelli, K. Refson, N. M. Harrison, and S. W. Cheong, Phys. Rev. Lett. 92, 056402 (2004).

[85] P. G. Radaelli, Y. Horibe, M. J. Gutmann, H. Ishibashi, C. H. Chen, R. M. Ibberson, Y. Koyama, Y. S. Hor, V. Kiryukhin, and S. W. Cheong, Nature (London) 416, 155 (2002).

[86] D. I. Khomskii and T. Mizokawa, Phys. Rev. Lett. 94, 156402 (2005).

[87] C. Stock, P. M. Gehring, H. Hiraka, I. Swainson, G. Xu, Z.-G. Ye, H. Luo, J.-F. Li, and D. Viehland, Phys. Rev. B 86, 104108 (2012).

[88] M. R. Suchomel, D. P. Shoemaker, L. Ribaud, M. C. Kemei, and R. Seshadri, Phys. Rev. B 86, 054406 (2012).

[89] T. Weber, Ph.D. thesis, Physikdepartment E21, Technische Universität München, Garching, Germany, 2016. 